THE ASTROPHYSICAL JOURNAL, 470:647-651, 1996 October 10

No copyright has been claimed for this article. Printed in U.S.A

\title{
ULTRAVIOLET JETS AND BRIGHT POINTS IN THE SOLAR CHROMOSPHERE. I. SEARCH FOR ONE-TO-ONE RELATIONSHIPS
}

\author{
J. W. Cook \\ E. O. Hulburt Center for Space Research, Code 7668, Naval Research Laboratory, Washington, DC 20375-5352 \\ R. J. RUTTEN \\ Sterrekundig Instituut, Postbus 80000, NL-3508 TA Utrecht, The Netherlands
}

AND

N. M. HOEKZEMA

Sterrekundig Instituut, Postbus 80000, NL-3508 TA Utrecht, The Netherlands

Received 1992 July 6; accepted 1996 April 2

\begin{abstract}
Ultraviolet spectrograms and spectroheliograms of the solar chromosphere are used to test the suggestion of Dere, Bartoe, \& Brueckner and Rutten \& Uitenbroek that bright points in quiet Sun cell inte-

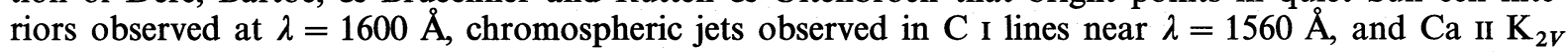
bright points are associated with each other and that they are all manifestations of the same wave interaction in the nonmagnetic chromosphere. We search for spatio-temporal connections between $1600 \AA$ bright points and $\mathrm{C}$ I blue jets using data from the High Resolution Telescope and Spectrograph VI rocket flight, comparing $1600 \AA$ spectroheliograms and a cospatial C I Doppler shift map on a pixel-bypixel basis. We find no direct evidence for spatial colocation of bright points and jets, not for instantaneous correspondence and also not when allowing for phase delays as long as 3 minutes. Also, the average brightness evolution and its rms fluctuation are not obviously different between sites of large $\mathrm{C}_{\mathrm{I}}$ blueshift and the remaining surface.
\end{abstract}

Subject headings: Sun: chromosphere - Sun: granulation - Sun: UV radiation

\section{INTRODUCTION}

Spectroheliograms from the NRL High Resolution Telescope and Spectrograph (HRTS) experiment covering a passband near $\lambda=1600 \AA$ (Cook, Brueckner, \& Bartoe 1983; Cook \& Ewing 1990, 1991) and similar filtergraph images taken with the Transition Region Camera (Bonnet et al. 1982; Foing \& Bonnet 1984) have mapped solar emission in a wavelength band where it arises primarily from the solar temperature minimum region. These images show quiet areas to be structured by the magnetic network, the latter outlined by clusters of closely packed bright points of arcsecond size that are long lived, at least relative to the duration of a 5 minute rocket flight. In addition, there are bright points within the internetwork areas or "cell interiors." They have similar size but evolve more rapidly, with characteristic lifetimes of about a minute. Whereas the network bright points are highly correlated with the absolute value of the magnetic flux density observed in photospheric magnetograms (Cook \& Ewing 1990), it is not known whether the internetwork bright points are similarly associated with the low-level concentrations of magnetic flux presumably present in internetwork areas.

The $1600 \AA$ internetwork bright points have often been assumed to be identical to the comparable $\mathrm{Ca} I \mathrm{II} \mathrm{K}_{2 V}$ grains because of their general morphological similarity (e.g., Bonnet et al. 1982; Cook et al. 1983; Foing \& Bonnet 1984; Dere, Bartoe, \& Brueckner 1986). The latter are internetwork bright points observed in a narrow band just blueward of the centers of the $\mathrm{Ca}$ II $\mathrm{H}$ and $\mathrm{K}$ lines. A magnetic origin has been claimed for these (Sivaraman \& Livingston 1982), but in their review of the extensive $K_{2 V}$ literature, Rutten \& Uitenbroek (1991a) concluded that $\mathrm{K}_{2 V}$ grains are of nonmagnetic hydrodynamical origin, with much pattern interference between different wave modes including vertical velocity oscillations that produce 5-10 $\mathrm{km} \mathrm{s}^{-1}$ sawtooth-shaped shifts of $\mathrm{Ca} \mathrm{II} \mathrm{K}_{3}$ line center. Rutten \& Uitenbroek (1991b) suggested a scenario in which $\mathrm{K}_{2 V}$ grains mark moments and sites where backfalling matter from a previous shock passage falls onto the next upcoming shock front, after a well-developed wave train has gone through sufficient cycles. The recent numerical simulations of Carlsson \& Stein (1994) quantify this scenario in detail (see reviews by Rutten 1994, 1995). A direct connection between $\mathrm{K}_{2 V}$ and $1600 \AA$ bright points is plausible, but so far, no $\mathrm{Ca}$ II $\mathrm{H}$ and $\mathrm{K}$ images of sufficient quality have been obtained simultaneously with $1600 \AA \AA$ images to establish such a relationship.

A separate phenomenon called "chromospheric jets" has been described by Dere, Bartoe, \& Brueckner (1983) and is also discussed in Dere et al. $(1984,1986)$. These jets are small sites, of roughly $1^{\prime \prime}$ extent, where the lines of the C I multiplet near $1561 \AA$ show bright, nonreversed emission peaks that are Doppler shifted over $10-20 \mathrm{~km} \mathrm{~s}^{-1}$, predominantly to the blue. Such Doppler shifts are large for the low chromosphere, and much larger than both the error in the standard of rest (based on narrow emission lines of Si I) and the typical measurement error per profile. Note that these jets differ from the higher velocity "explosive events" seen in C IV lines from higher layers (see Dere et al. 1991; Dere 1994 and references therein). Jets occur primarily in internetwork areas; the average jet lifetime is around $40 \mathrm{~s}$. At first, Dere et al. (1983) interpreted these jets as disk representations of off-limb white-light spicules, but Dere et al. (1986) suggested instead that they are aspects of the same phenomenon which produces internetwork $1600 \AA$ bright points and, presumably, $\mathrm{Ca}$ II $\mathrm{K}_{2 V}$ grains. Rutten \& Uitenbroek (1991a, 1991b) then speculated that if this is the case, the jets should represent the $\mathrm{Ca}$ II $\mathrm{K}_{3}$ oscillation at another 
height and phase, so that spatio-temporal correlations should exist between $C_{I}$ jets and $1600 \AA$ bright points when phase delays up to a few minutes are admitted.

In this paper, we search for such time-lagged correlations on a pixel-by-pixel basis but do not find clear evidence for such a relationship. In a companion paper (Hoekzema, Rutten, \& Cook 1996), we turn to statistical methods averaging over many pixels; weak but significant relationships then emerge.

\section{INSTRUMENT AND OBSERVATIONS}

HRTS has flown nine times as a sounding rocket experiment, and also on the Spacelab 2 mission onboard the Space Shuttle. In this paper we analyze data from the HRTS VI rocket flight above White Sands on 1988 November 20.

The basic HRTS instrument has been described by Brueckner, Bartoe, \& VanHoosier (1978) and Brueckner \& Bartoe (1983). HRTS VI consisted of a $30 \mathrm{~cm}$ Cassegrain telescope which fed a stigmatic tandem-Wadsworth slit spectrograph (Bartoe \& Brueckner 1975) covering a wavelength range of $1520-1580 \AA$, a broadband slit-jaw spectroheliograph tuned to a $10 \AA$ wide passband centered at 1600 $\AA$, and an $\mathrm{H} \alpha$ slit-jaw imager. The solar target area included quiet regions, a coronal hole, and several small active regions without sunspots. It was imaged with the broadband spectroheliograph and scanned with the spectrograph by stepping the slit transversely across the image. Both the spectroheliograph images and the spectra were recorded on film and digitized later with a densitometer.

The observing program and the data have been described by Dere et al. $(1989,1991)$ in the context of coronal holes and explosive events. In this paper, we use seven sequential $1600 \AA$ images and a cospatial Doppler shift map obtained from the spectrograph data for a selected area near disk center.

\subsection{The $1600 \AA$ Images}

The broadband spectroheliograph produced an image of approximately $7.5 \times 15 \mathrm{arcmin}^{2}$ field size from a reflection off the spectrograph slit. According to standard modeling of the solar atmosphere (Vernazza, Avrett, \& Loeser 1981), $70 \%$ of the flux in its $10 \AA$ passband around $1600 \AA$ arises from the solar temperature minimum continuum with the remainder contributed by chromospheric emission lines within the passband. Images of various exposure times were obtained during the HRTS VI flight; we use the $7.2 \mathrm{~s}$ exposures here because these show internetwork structure best. There are eight such exposures covering the area of interest scanned by the spectrograph slit, respectively taken at $90 \mathrm{~s}$, 199 s, 221 s, 242 s, 274 s, 295 s, 335 s, and 357 s after the moment of the HRTS VI launch. The first exposure is from early in the flight; the pointing was later changed before the spectrograph raster sequences were executed that are analyzed here. We have therefore not used this initial exposure except for visual inspections. The intervals between the remaining images are about $22 \mathrm{~s}$, except after the fourth and sixth, when a change of pointing was made to start a new spectrograph raster sequence. The image taken at $221 \mathrm{~s}$ is shown in the top panel of Figure 1 (Pl. 18). A subfield of all seven images is shown in Figure 3.

The $1600 \AA$ images were calibrated following Cook \& Ewing (1990). The microdensitometer output was converted into relative intensities using the film characteristic curve and the average intensity in each image was then set to 150 ergs $\mathrm{cm}^{-2} \mathrm{~s}^{-1} \AA^{-1} \mathrm{sr}^{-1}$, corresponding to a disk center continuum intensity at $1600 \AA$ of 170 ergs cm $\mathrm{cm}^{-2} \mathrm{~s}^{-1} \AA^{-1}$ $\mathrm{sr}^{-1}$ (Cook, Brueckner, \& VanHoosier 1980) and a centerto-limb ratio of 0.885 (Samain 1979) for the viewing angle of the center of the area studied below $(\cos \theta=\mu=0.836)$. Each intensity value in the images was then converted to brightness temperature. The error in this calibration is approximately $(+67$; -92$) \mathrm{K}$ in brightness temperature. However, for the present study the differential excursions from the average brightness temperature per spatial position are more important. For these, the primary error source is the inherent noise from film grain, which is less than $20 \mathrm{~K}$ in brightness temperature.

\subsection{The Spectrograph Maps}

The length of the spectrograph slit was 920", approximately a solar radius. The slit was widened from its usual 0 ..5 width to 1.0 in order to increase the speed of the spectrograph and scan as large an area as possible during the short flight, at the cost of spectral resolution $(\Delta \lambda=0.10$ $\AA$ ). The slit was stepped across the target area in four raster sequences (shown in Fig. 1 of Dere et al. 1989). The first is separated from the other three by $2^{\prime}$ and is not used in this paper. The other three sequences are adjacent; together, they cover an area of $920^{\prime \prime}$ along the slit by $107^{\prime \prime}$ in the stepping direction. In the first raster, the slit was stepped over $50^{\prime \prime}$ in 26 steps of $2^{\prime \prime}$; the second covered $20^{\prime \prime}$ in 21 steps of $1^{\prime \prime}$; the third covered $40^{\prime \prime}$ in 21 steps of $2^{\prime \prime}$ width. From these, geometrically correct maps were produced by applying small corrections for actual slit positions and for the small gaps between the sequences.

The spectrograms cover the 1520-1580 $\AA$ wavelength range and contain the Si II $1526 \AA$ and $1533 \AA$ lines, the C IV

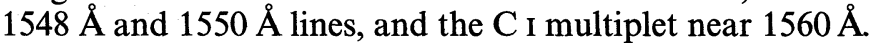
After scanning, the digitized spectra were converted to absolute intensity using the film characteristic curve and a comparison between the relative intensities so obtained with absolute intensities from previous HRTS calibrations. The accuracy of the absolute calibration is not critical to the analysis reported here.

Following standard HRTS reduction procedures (Dere et al. 1984), maps of the intensity and velocity from a spectral line were constructed for the area covered by the raster sequences by measuring the zeroth moment (line intensity) and the first moment (average wavelength position) of a spectral line profile for each spatial resolution element along the slit for each slit position. The areas between raster steps are filled in through an interpolation scheme. This procedure requires relative intensity calibration only. The wavelength scale for converting first moments into Doppler shifts is obtained from the narrow $\mathrm{Si}$ I lines within the spectral range.

For the present analysis we have produced intensity and Doppler shift maps for the C I multiplet near $1560 \AA$ by summing the intensities and averaging the Doppler shifts of the two composite $C_{\text {I }}$ profiles at $1560.7 \AA$ and $1561.4 \AA$. The statistical Doppler shift measurement error has been estimated by Dere et al. (1984) to be about $2 \mathrm{~km} \mathrm{~s}^{-1}$. However, there are several possible systematic errors. The C I multiplet actually consists of six components shown in Fig. 3 of Dere et al. (1983). The unblended $1560.31 \AA$ component is usually weak and is not used in our map construction, while the other five components form two blended 
profiles at $1560.7 \AA$ (composed of $1560.68 \AA$ and $1560.71 \AA$ ) and at $1561.4 \AA$ (composed of $1561.34 \AA$ and $1561.44 \AA$ ). In addition, the Doppler shifts determined from the two observed profiles tend to differ because the $C_{\text {I }}$ lines are optically thick. Therefore, we are not certain that our firstmoment C I Doppler shift determinations actually represent true bulk flows in the chromosphere. Nevertheless, they are the only velocity indicators in hand, and we are also studying Doppler shifts here of relatively large magnitude.

Locations for which the first moment indicates Doppler shifts in excess of $25 \mathrm{~km} \mathrm{~s}^{-1}$ have been set to this limiting value in our map construction. Locations where the $\mathrm{C}_{\mathrm{I}}$ intensities do not allow for accurate determination of the line profile are left dark. This occurs typically because of weak emission, but occasionally because of film saturation from exceptionally bright emission.

\subsection{Coregistration of Images and Maps}

The spectrograph slit is visible on the spectroheliograph images, so that its position at the mid-moment of each spectroheliograph exposure could be determined and the C I maps coregistered with the $1600 \AA$ images. The final maps and images have been digitized at 0.5 pixel size. Their coregistration can be checked by direct frame comparison using an image display system. We estimate the registration to be accurate to 3 pixels, or 1".5.

A subfield of $150 \times 87 \mathrm{arcsec}^{2}$ size has been selected from the complete $920 \times 107 \operatorname{arcsec}^{2}$ map for more detailed analysis described below. It avoids larger scale image imperfections in the $\mathrm{C}_{\mathrm{I}}$ velocity map (fiducial wire positions, dust on the slit, and extended areas where the $\mathrm{C}_{\mathrm{I}}$ intensity did not allow reliable velocity determination) and is covered by all seven co-aligned $1600 \AA$ images. Its center has solar position angle $\mu=0.836$. A smaller subarea of $50 \times 50 \operatorname{arcsec}^{2}$ is also used below.

\section{RESULTS}

We search for correspondence between internetwork bright points in the $1600 \AA$ images and sites of chromospheric jets on the $\mathrm{C}_{\mathrm{I}}$ velocity map. The input data are illustrated in Figure 1 in the form of three co-aligned panels, respectively a part of the $1600 \AA \AA$ image taken at $t=221 \mathrm{~s}$, the corresponding part of the $\mathrm{C}_{\mathrm{I}}$ intensity map, and the corresponding part of the $\mathrm{C}_{\mathrm{I}}$ velocity map. The agreement in bright network patterning between the $1600 \AA$ image (top) and the $\mathrm{C}_{\mathrm{I}}$ intensity map, (middle) is excellent. Sites of large upward (blue) and downward (red) motion in the C I Doppler shift map are predominantly located in the internetwork regions. Within these, large Doppler shift sites appear to be randomly distributed.

For a closer look, we show in Figure 2 (Pl. 19) the result of summing the seven individual $1600 \AA$ images for the $150 \times 87 \operatorname{arcsec}^{2}$ subfield. This area lies just left of the fiducial line in the middle of the top panel of Figure 1. The long-lived network features are enhanced in this $158 \mathrm{~s} \mathrm{sum-}$ mation, whereas the rapidly varying internetwork brightness features are suppressed. In addition Figure 2 displays the positions of "blue jets" superposed as green pixels. Blue jets are here defined as locations with $\mathrm{C}_{\mathrm{I}}$ blueshift in the range $15-25 \mathrm{~km} \mathrm{~s}^{-1}$. Dere et al. (1983) specified a velocity range of $10-20 \mathrm{~km} \mathrm{~s}^{-1}$ for chromospheric jets, but after examining several trial velocity ranges, we settled on 15-25 $\mathrm{km} \mathrm{s}^{-1}$ because this range preserves jets as relatively pointlike features, results in somewhat less crowded area cover- age, gives a larger degree of confidence in the velocities as being physically meaningful, and includes the strongest and most obvious examples of chromospheric jets. In addition, we look only at blueshifts although Dere et al. (1983) also described a few redshifted features as chromospheric jets. Figure 2 confirms that blue jets are generally found in internetwork regions (dark red), avoiding the chromospheric network (bright) - although there are exceptions, some blue jets being located within the bright elements of the network. The distribution of blue jet locations over the internetwork regions again seems random, perhaps with some mesoscale clustering.

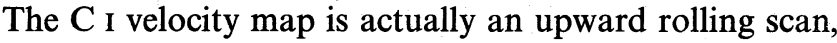
from bottom to top in the lower panel of Figure 1, sampling the chromospheric velocity field in time-dependent fashion. Each horizontal pixel row was produced from one spectrogram, the next higher row from the subsequent spectrogram, etc. In contrast, the $1600 \AA \AA$ spectroheliograph frames are true images, covering the entire field at once during exposure. Thus, the question we may investigate is whether, at the site of a chromospheric jet that is observed only at the single moment when the spectrograph rastering sampled that location, a concurrent cospatial internetwork bright point occurs in the $1600 \AA$ images, possibly with some phase delay in temporal development. The scenario of Rutten \& Uitenbroek (1991b) suggests that a bright point should develop a few minutes after the initial upwardmoving chromospheric jet. The data permit searching for such a correlation with delays up to $174 \mathrm{~s}$, which is the interval between the moment at which the slit rastering started (186 s after launch, bottom line in C I panels of Fig. 1) and the moment of exposure of the last $1600 \AA \AA$ image (360 s after launch).

We first searched for easy-to-note correspondence by visually comparing, on an image display monitor, the Doppler shift map with a movie rendition of the $1600 \mathrm{~A}$ images. After noting the site of a blue jet, we watched the temporal development of the $1600 \AA$ brightness at this location. Doing so for a number of jet sites, we did not note any directly obvious relationship between blue jet locations and $1600 \AA$ bright points, also not when allowing for temporal phase differences of either sign.

Figure 3 (Pl. 20) enables the reader to simulate such direct inspection. It displays a yet smaller subfield of $50 \times 50$ $\operatorname{arcsec}^{2}$; the larger scale is chosen to keep a particular jet location easily identifiable between the seven images while the area is yet large enough to contain several network cells. This subfield lies to the lower right in the left-hand half of Figure 2. The superposed green pixels again mark 15-25 $\mathrm{km} \mathrm{s}^{-1}$ blueshifts; they are the same for each image. The subfield was scanned bottom to top by the slit spectrograph from $t=200$ to $t=240 \mathrm{~s}$ after launch. Therefore, the upper left image displays $1600 \AA$ brightness at the moment when the jets near the bottom were sampled, while the lower left image was taken simultaneously with the jet sampling near the top of the field; the seven image sequence covers possible phase lags from $-41 \mathrm{~s}$ to $+157 \mathrm{~s}$.

Inspection shows no direct relationship between blue jets and $1600 \AA$ bright points. A few jets lie in the bright area just above the center of the field, but this is part of the network as is obvious from Figure 2. If anything, there seems not to be coincidence between jets and above-average

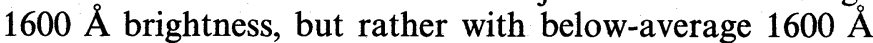
darkness at a phase lag of about $20 \mathrm{~s}$ : the nonnetwork green 
pixels overlying the bottom of the $t=221 \mathrm{~s}$ image, the middle of the $t=242 \mathrm{~s}$ image, and the top of the $t=274 \mathrm{~s}$ image seem to preferentially lie in dark areas.

In order to perform a more quantitative search for relationships between chromospheric jets and $1600 \AA \AA$ bright points, we now examine the $1600 \AA$ brightness characteristics applying a separation between blue jet locations (sites with $C_{\text {I }}$ blueshifted over $15-25 \mathrm{~km} \mathrm{~s}^{-1}$ at the moment of Doppler shift sampling by the spectrograph) and the remaining areas, including network. We call these the blue and the nonblue areas, respectively. The area statistics are shown in Figure 4 which displays the Doppler shift distribution for the $150 \times 87 \mathrm{arcsec}^{2}$ subfield in the form of a histogram with $5 \mathrm{~km} \mathrm{~s}^{-1}$ bins, normalized such that the pixels for which the velocity could not be measured are not included. Approximately $14 \%$ of this subfield consists of blue areas (the two left most columns of the histogram), whereas the corresponding redshift bins on the right contain only $5 \%$ of the total. This difference illustrates the preponderence of blue over red jets in Figure 1, as already noted by Dere et al. (1983).

Figure 5 shows the spatially averaged brightness temperature of each $1600 \AA$ image, split between blue and

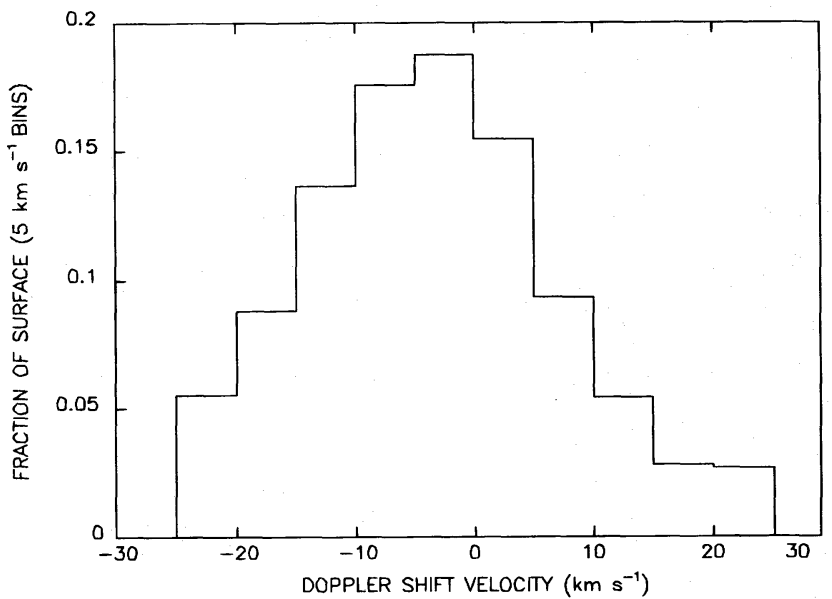

FIG. 4.-Histogram distribution of velocities within the $150 \times 87$ $\operatorname{arcsec}^{2}$ subfield. Blue jets (15-25 km s${ }^{-1}$ blueshift) cover $14 \%$ of the surface.

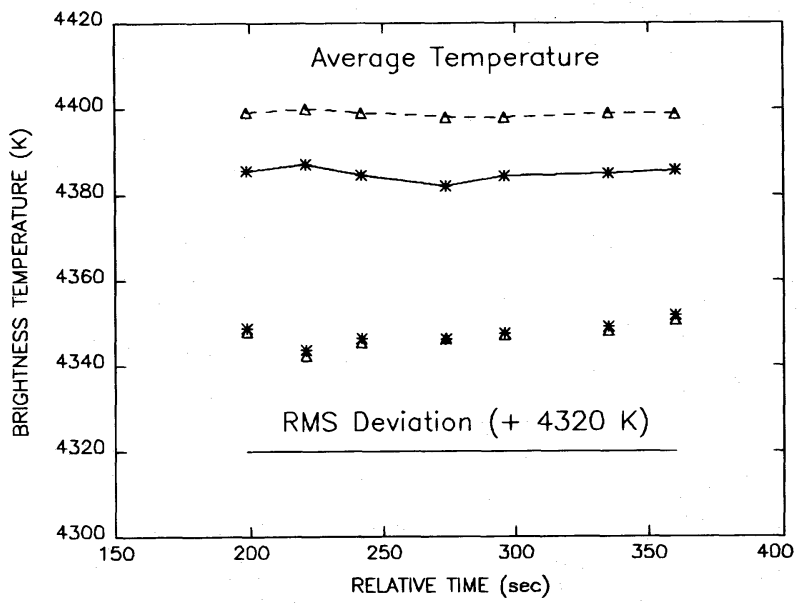

FIG. 5.-Upper symbols and curves: spatially averaged brightness temperature for the $150 \times 87 \operatorname{arcsec}^{2}$ subfield of each of the seven $1600 \AA$ images, split between blue (asterisks) and nonblue (triangle) areas. Lower symbols: blue and nonblue spatial rms deviations from average, at an offset of $4320 \mathrm{~K}$ above zero. nonblue areas (solid and dashed curves). Since the average intensity of each full-field image was set equal to 150 ergs $\mathrm{cm}^{-2} \mathrm{~s}^{-1} \AA^{-1} \mathrm{sr}^{-1}$, the slight variations in average temperature reflect small differences in the intensity distribution between the full-field and subfield images, and also yet smaller differences between the blue versus the nonblue distributions per image. The average temperature is higher in the nonblue area which includes the bright network; blue areas occur predominantly in cell centers.

The symbols in the lower half of Figure 5 measure corresponding rms variations. For the blue and nonblue areas of each image, respectively, they specify the rms deviation of the brightness temperature per pixel from the time-averaged value of that pixel plotted in Figure 2. These spatial rms deviations are very similar for the blue and nonblue areas; they measure approximately $25 \mathrm{~K}$ with a slight excess for the blue areas (asterisks).

In Figure 6 we show the development of the average brightness temperature for seven horizontal strips, each measuring $150 \times 12.5 \operatorname{arcsec}^{2}$, which together cover the $150 \times 87 \operatorname{arcsec}^{2}$ subfield. These were consecutively sampled by the spectrograph slit, lengthwise from bottom to top. Each of the seven curves is shifted in relative time so that the moment at which the slit sampled the middle of each strip occurs at $t=0 \mathrm{~s}$. Thus, the seven strips are lined up in common phase with respect to the Doppler shift measurement, while each individual curve covers a different temporal range around that moment. This display serves to search for a systematic phase advance or phase delay in the occurrence of bright points at sites of chromospheric jets. Each curve plots the difference between the brightness temperature averaged per image over the strip and the timeaveraged mean of that strip in all seven images, again split between blue and nonblue pixels. Across a strip, the Doppler shift measurements all occur within about $20 \mathrm{~s}$, a period shorter than the 1 minute lifetime of bright points and the $40 \mathrm{~s}$ duration of jets measured by Dere et al. (1983). On the other hand, the strips are sufficiently long that small-scale brightness variations from film noise and from actual solar brightness modulation such as the $250 \mathrm{~s}$ brightness oscillation with $50 \mathrm{~K}$ average amplitude reported by

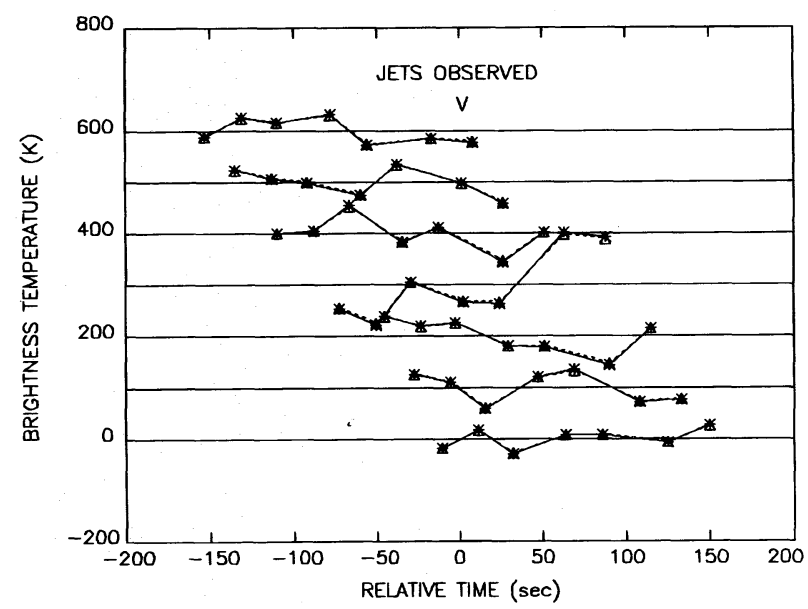

Fig. 6.-Brightness temperature evolution for seven 12".5 wide strips across the $150 \times 87 \operatorname{arcsec}^{2}$ subfield, each on a shifted temporal axis so that $t=0$ corresponds to the moment of midstrip sampling by the spectrograph slit for all curves. The symbols specify the average difference in brightness temperature between each strip and its seven-image average, respectively for blue areas (asterisks) and nonblue areas (triangles). The curves are displaced vertically from each other by $100 \mathrm{~K}$. 


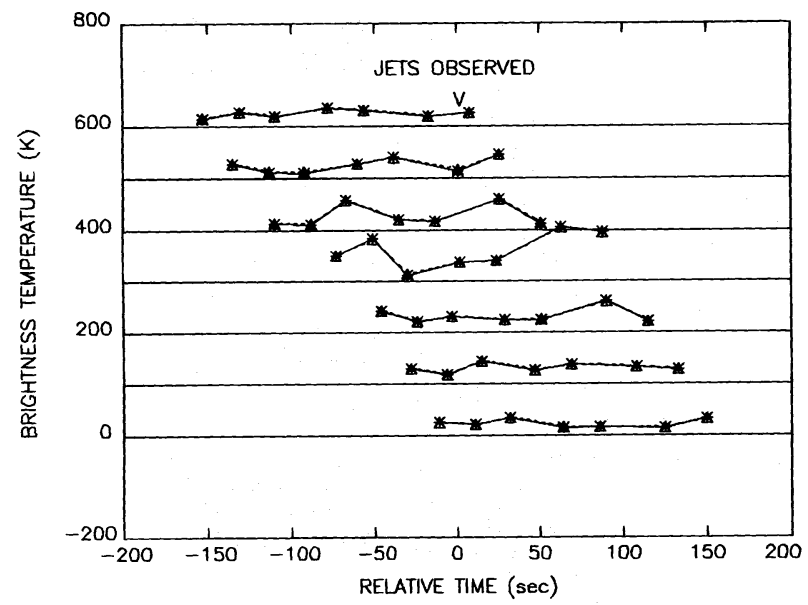

Fig. 7.-As Fig. 6 for rms brightness variation

Cook \& Ewing (1991) tend to cancel out when averaged over a full strip. But if $1600 \AA$ bright points appear at jet sites with a characteristic phase shift prior to or after the jet occurs, they should not cancel out but add constructively and so produce an increase in the average brightness temperature at jet sites at that characteristic phase shift. An average brightness peak should then be present at a given time interval before or after $t=0$ in Figure 6 for blue areas, while such a peak should not be present in the nonblue data.

How large should such a differential peak be? An average network bright point is approximately $300 \mathrm{~K}$ brighter than the average brightness temperature (Cook \& Ewing 1990). The internetwork bright points are typically not this bright but still produce a signal of order $50-100 \mathrm{~K}$ (Foing \& Bonnet 1984), well above the average background variation. Thus a systematic brightening of order $100 \mathrm{~K}$ in the bluearea temporal evolution relative to the nonblue evolution in Figure 6 would represent evidence for bright-point emission at that particular phase delay relative to the jet occurrence. However, the curves in Figure 6 show modulation due to differences between the normalization of the large-field original images and the strip-wise averaging, but no signifcant difference between the blue and nonblue areas. Their brightness temperatures vary in step, with only the slight (order $10 \mathrm{~K}$ ) deficits in blue areas also seen in Figure 5. There is no sign of any systematic blue over nonblue brightening of order $100 \mathrm{~K}$ at any phase shift. In Figure 7 we show a similar plot for the average rms deviation in brightness temperature per strip. Again we find no systematic difference between blue and nonblue areas.

\section{DISCUSSION}

We have been unable to find any systematic correlation between the sites of $1600 \AA$ internetwork bright points and sites of blue jets, also not when allowing for phase delays between the two phenomena as long as 3 minutes. Also, we have not found any systematic difference in the temporal evolution of the average brightness or its average rms fluctuations between blueshifted areas and the remaining nonblueshifted area. Thus we find no clear evidence for a direct relationship between internetwork bright points and jets as suggested by Rutten \& Uitenbroek (1991a, 1991b). A more intricate and indirect relationship, derived from statistical analysis of the same data, is described by Hoekzema et al. (1996).

Our analysis above does not rule out a direct correlation for phase delays above 3 minutes, which may occur in Rutten \& Uitenbroek's scenario if the wake oscillation requires multiple cycles before shocks of sufficient strength develop. Unfortunately, such ranges of temporal development cannot be investigated from a sounding rocket flight.

A desirable option for further study is to employ groundbased Ca II data over a longer time span, in order to compare $\mathrm{Ca}$ II $\mathrm{K}_{2 V}$ bright points with rocket maps of chromospheric Doppler shifts as used here. This may also directly confirm (or disprove) the identity of $\mathrm{Ca}$ II $\mathrm{K}_{2 V}$ bright points and $1600 \AA$ bright points, but requires a HRTS rocket flight with simultaneous high-quality groundbased coverage in $\mathrm{Ca}$ II. So far this has not been successfully accomplished. Another option, obviously, is to obtain space observations from platforms in orbit. More generally, such studies of chromospheric internetwork dynamics may supply a valuable probe of the magnetic canopies which are thought to occur over the interiors of supergranulation cells.

J. W. Cook's work was supported by NASA under DPR W-14,541 and by the Office of Naval Research. N. M. Hoekzema's research was supported by the Netherlands Foundation for Research in Astronomy (NFRA) with financial aid from the Netherlands Organization for Scientific Research (NWO).

\section{REFERENCES}

Bartoe, J.-D. F., \& Brueckner, G. E. 1975, J. Opt. Soc. Am., 65, 13

Bonnet, R. M., Bruner, M., Acton, L. W., Brown, W. A., Decaudin, M., \&

Foing, B. 1982, A\&A, 111, 125

Brueckner, G. E., \& Bartoe, J.-D. F. 1983, ApJ, 272, 329

Brueckner, G. E., Bartoe, J.-D. F., \& VanHoosier, M. E. 1978, in Proc. OSO-8 Workshop, ed. E. Hansen \& S. Schaffner (Boulder: Colorado), 380

Carlsson, M., \& Stein, R. F. 1994, in Chromospheric Dynamics, Proc.

Miniworkshop, ed. M. Carlsson (Oslo: Inst. Theor. Astrophys.), 47

Cook, J. W., Brueckner, G. E., \& Bartoe, J.-D. F. 1983, ApJ, 270, L89

Cook, J. W., Brueckner, G. E., \& VanHoosier, M. E. 1980, J. Geophys. Res. A5, 85, 2257

Cook, J. W., \& Ewing, J. A. 1990, ApJ, 355, 719

1991, ApJ, 371, 804

Dere, K. P. 1994, Space Sci. Rev., 70, 21

Dere, K. P., Bartoe, J.-D. F., \& Brueckner, G. E. 1983, ApJ, 267, L65

1984, ApJ, 281, 870
-1986, ApJ, 305, 947
Dere, K. P., Bartoe, J.-D. F., Brueckner, G. E., Ewing, J., \& Lund, P. 1991, J. Geophys. Res. A6, 96, 9399

Dere, K. P., Bartoe, J.-D. F., Brueckner, G. E., \& Recely, F. 1989, ApJ, 345, L95

Foing, B., \& Bonnet, R. M. 1984, ApJ, 279, 848

Hoekzema, N. M., Rutten, R. J., \& Cook, J. W. 1996, ApJ, submitted

Rutten, R. J. 1994, in Chromospheric Dynamics, Proc. Miniworkshop, ed. M. Carlsson (Oslo: Inst. Theor. Astrophys.), 25

. 1995, in Helioseismology, Proc. Fourth SOHO Workshop ed. J. T. Hoekzema, V. Domingo, B. Fleck, \& B. Battrick (ESA SP-376 Vol. 1) (Noordwijk: ESTEC), 151

Rutten, R. J., \& Uitenbroek, H. 1991a, Solar Phys., 134, 15

. 1991b in Mechanisms of Chromospheric and Coronal Heating, ed. P. Ulmschneider, E. R. Priest, \& R. Rosner (Berlin: Springer), 48 Samain, D. 1979, A\&A, 74, 225

Sivaraman, K. R., \& Livingston, W. C. 1982, Solar Phys., 80, 227

Vernazza, J. E., Avrett, E. H., \& Loeser, R. 1981, ApJS, 45, 635 


\section{PLATE 18}

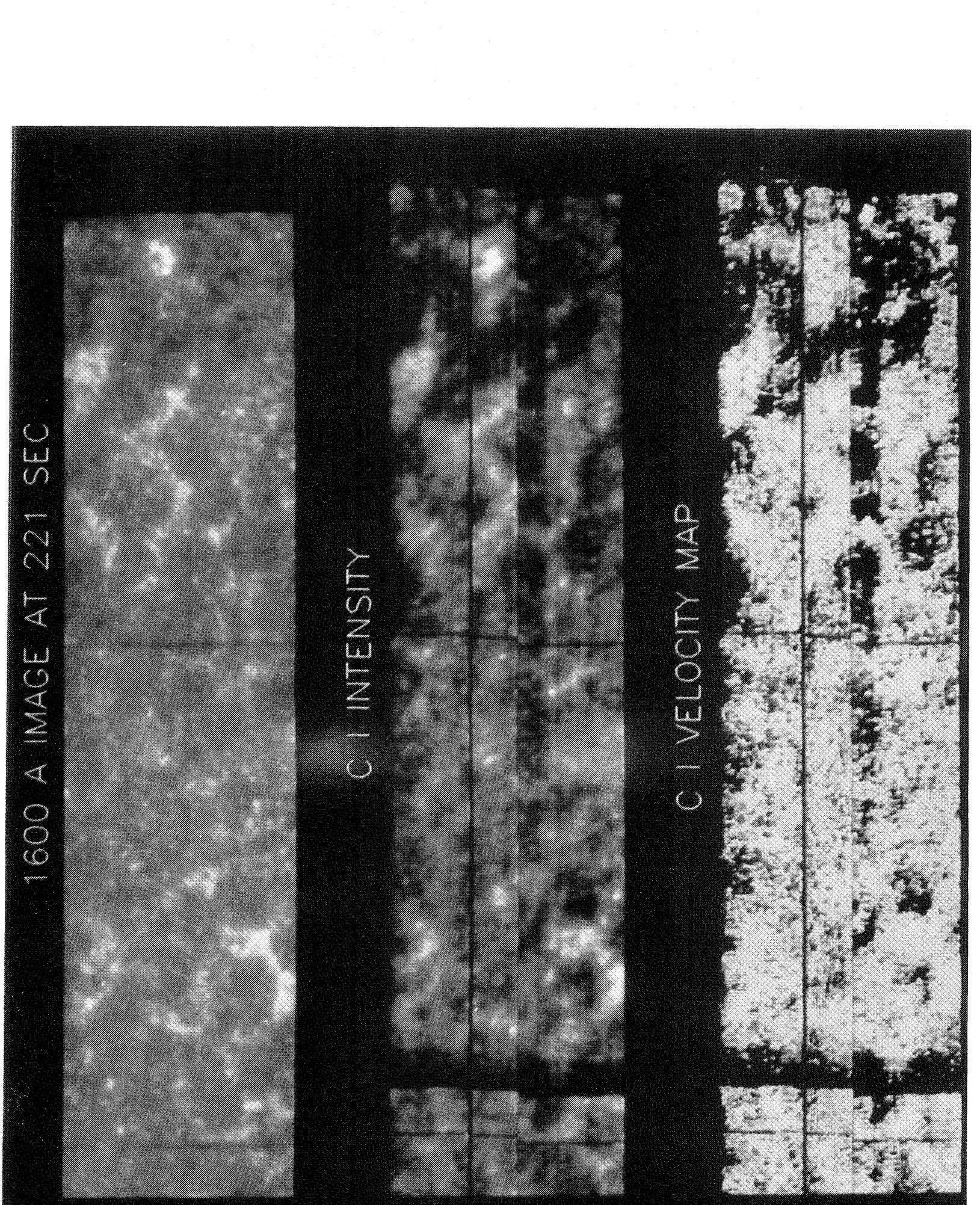

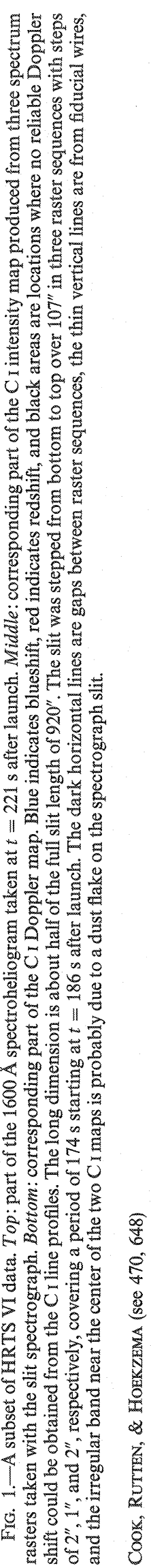




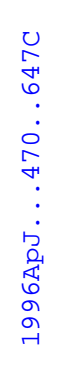
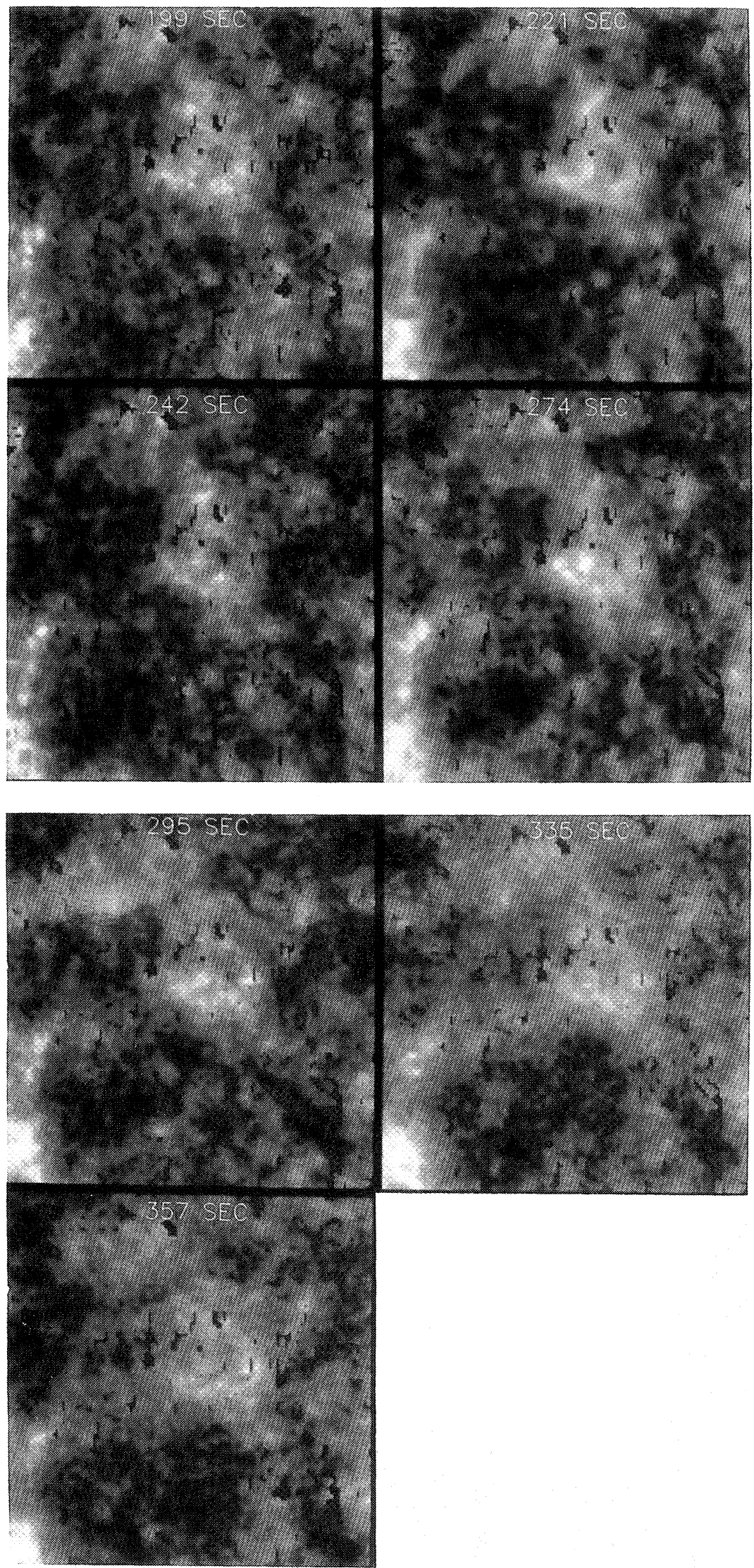

Fig. 3. - The $50 \times 50 \operatorname{arcsec}^{2}$ subfield in the sequence of seven $1600 \AA$ images. The subfield lies to the lower left from the center of Fig. 2 . The times of exposure (seconds after launch) are indicated. The green pixels again identify blue jet locations as sampled by the spectrograph. The spectrograph slit scanned this subfield from bottom to top during $t=200 \mathrm{~s}$ to $t=240 \mathrm{~s}$.

CoOK, Rutten, \& Hokkzema (see 470,649 ) 